\title{
Jump to Contact, Neck Formation, and Surface Melting in the Scanning Tunneling Microscope
}

\author{
L. Kuipers and J. W. M. Frenken \\ FOM-Institute for Atomic and Molecular Physics, Kruislaan 407, 1098 SJ Amsterdam, The Netherlands
}

(Received 11 January 1993)

\begin{abstract}
With a scanning tunneling microscope the $\mathrm{Pb}(110)$ surface was studied from room temperature to temperatures where surface melting occurs. At room temperature scanning tunneling microscopy images of $\mathrm{Pb}(110)$ can be recorded with atomic resolution. At higher temperatures we observe a jump to contact by the surface, resulting in the formation of a connecting neck of $\mathrm{Pb}$ between the surface and the tip. As the tip is retracted, the neck elongates and finally breaks. The dependence of the average neck height just before rupture on temperature and retraction speed suggests that mobile adatoms are responsible for the growth of the neck.
\end{abstract}

PACS numbers: $61.16 . \mathrm{Ch}, 68.35 . \mathrm{Fx}, 68.35 . \mathrm{Gy}, 68.70 .+\mathrm{w}$

The adhesion of two solid bodies is of great relevance to the understanding of the fundamental physics of sintering, wear, and friction. Since the introduction of the scanning tunneling microscope (STM) [1] and the atomic force microscope (AFM) [2], which are able to address local surface properties on the atomic scale, the interaction between the probing tip and the surface has received much attention $[3,4]$. Pethica and Sutton have suggested that the tip and surface might suddenly jump to produce mechanical contact when they are brought together close enough [3]. Recently, molecular dynamics simulations have shown that if either the tip or the surface material tends to wet the other, jumps over considerable distances may be observed [5]. The jump can be followed by a rapid growth of the connecting neck [6]. The jump distance increases with increasing density of adatoms on the surface, resulting in very large jumps $(>10$ $\AA$ ) for surfaces that are melted [7,8].

In this Letter we report such a neck formation during temperature-dependent STM measurements on $\mathrm{Pb}(110)$. This surface is known for its surface-melting behavior [9] and its correspondingly high self-diffusion coefficient [10]. At elevated temperatures we systematically observe a jump to contact followed by the subsequent buildup of a sizable neck. The process depends strongly on the surface temperature. We propose that mobile adatoms are responsible for the formation of the neck.

The experiments were performed in ultrahigh vacuum ( $p<1 \times 10^{-10}$ mbar) using an STM designed especially for use at high temperatures. This instrument has demonstrated atomic resolution on various metal and semiconductor surfaces up to $750 \mathrm{~K}$. The STM tip was prepared by electrochemical etching of a $0.25 \mathrm{~mm}$ diam W wire followed by annealing in vacuum. The tip was further prepared in situ by field electron and field ion emission. The $\mathrm{Pb}$ sample was spark-cut from a singlecrystal ingot, mechanically polished, and chemically etched. It was cleaned in ultrahigh vacuum by $\mathrm{Ar}$ ion sputtering at $400 \mathrm{~K}$. Surface cleanliness and crystalline order were checked with Auger-electron spectroscopy and low-energy electron diffraction. By radiatively heating the rear side of the crystal temperatures up to the melting point $\left(T_{m}=600.7 \mathrm{~K}\right)$ were obtained. The temperature was monitored with an infrared pyrometer (Ircon model 6000) and a Chromel-Alumel thermocouple connected directly to the sample.

At room temperature, stable images of $\mathrm{Pb}(110)$ were obtained with atomic resolution, as illustrated by the surface image in Fig. 1. The close-packed rows along the [110] direction, as well as the atoms within the rows, are clearly resolved. The peak-to-valley corrugation amplitude is $0.26 \AA$ along the [001] direction and $0.18 \AA$ along the $[1 \overline{1} 0]$ direction. The image contains two monatomic steps on the left-hand side. The highly dynamic character of the $\mathrm{Pb}(110)$ surface even at room temperature is illustrated by two artifacts in the image. First, the two steps on the left-hand side are rugged instead of straight, probably as a result of kink diffusion along the steps

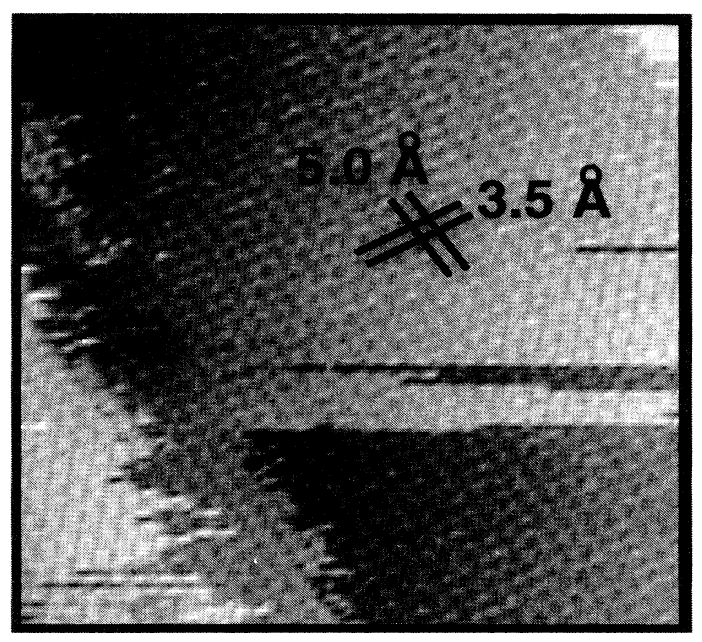

FIG. 1. Grey scale representation of an STM image of the $\mathrm{Pb}(110)$ surface at room temperature $(131 \AA \times 128 \AA$, $\left.V_{t}=+230 \mathrm{mV}, I_{t}=1.0 \mathrm{nA}\right)$. The grid indicates the [1 10$]$ and the [001] directions. The numbers indicate the distance between atoms in angstroms. 
$[11,12]$. Second, the sharp, horizontal steplike features near the middle of the image are not topographical steps on the surface, but instead reflect changes of the local height of the surface by a single atomic plane within the duration of one scan line $(\approx 0.5 \mathrm{~s})$.

However, at sample temperatures only slightly above room temperature, regular tunneling was frequently interrupted by short circuits between the surface and the tip. With increasing temperature the maximum time of normal tunneling before the short circuit decreased rapidly. At $318 \mathrm{~K}$ it was $240 \mathrm{~s}$ maximum, at $332 \mathrm{~K}, 36 \mathrm{~s}$, and at $350 \mathrm{~K}$, as short as $5 \mathrm{~s}$ (tunneling with a current of 2 $\mathrm{nA}$ at a sample voltage of $-5.0 \mathrm{~V}$ ). At even higher sample temperatures our system could not detect any tunneling current $\left(I_{t}<2.5 \mathrm{pA}\right)$ prior to a short circuit. Regular tunneling with sample voltage magnitudes below $0.5 \mathrm{~V}$ was not possible at and above $318 \mathrm{~K}$, without a short circuit occurring immediately. In all cases the change in the measured current took place faster than the time resolution of the measurements $(120 \mu \mathrm{s})$.

Surprisingly, once formed, the short circuit could persist even during tip retractions of up to several thousand angstroms. The height of retraction at which the short circuit disappeared $(L)$ was found to depend on the retraction speed and on the temperature of the sample. Figure 2 shows that $L$ increased with increasing temperature and with decreasing tip-retraction speed. In the limit of zero retraction speed $L$ appears to diverge for each temperature. Figure 2 also shows that an increase in temperature of merely $20 \mathrm{~K}$ (from 331 to $350 \mathrm{~K}$ ) led to a tripling of $L$. At temperatures above $450 \mathrm{~K}$, where $\mathrm{Pb}(110)$ was surface melted [9], the short circuits remained intact over the entire retraction range of 1.0 $\mu \mathrm{m}$ even for the highest retraction speeds. Then a coarse

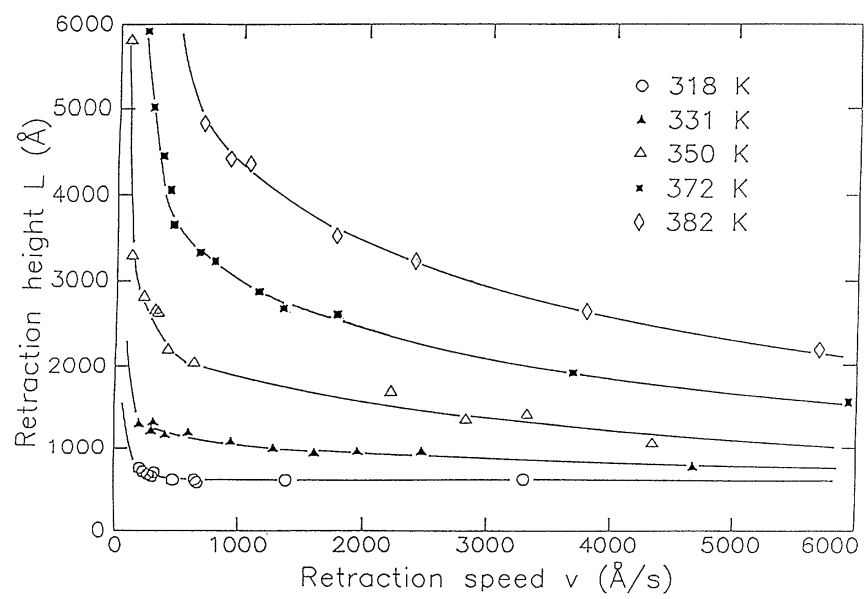

FIG. 2. Average retraction height at which the short circuit disappears as a function of tip-retraction speed for different temperatures (initial sample voltage $-5 \mathrm{~V}$, short circuit current $23 \mathrm{nA}$ ). Individual heights lie within $20 \%$ of the average. The solid curves serve to guide the eye. mechanical retraction was needed to break the connection. In all cases $L$ was independent of the sign of the short circuit current. It did, however, depend somewhat on the magnitude of the short circuit current, the highest current of $\approx 23 \mathrm{nA}$ leading to a $450 \AA$ increase in $L$. During the short circuit, the voltage drop over the tipsurface junction reduced to practically zero $(<1 \mathrm{mV})$ and the short circuit current was determined by the input impedance of the current preamplifier and the original sample voltage. The resistance of the tip-surface junction was less than the minimum value of $10 \mathrm{k} \Omega$ that could be measured with the STM.

Each time that the short circuit was broken the tip approached the surface again. When the approach was slow, the tip "found" the surface always at a height that was within $200 \AA$ of the height at which the short circuit had initially occurred. The cycle of approach, short circuit, and breaking of the electrical contact repeated itself more or less periodically. Typically, such cycles took between $0.5 \mathrm{~s}$ for the smallest retractions and $100 \mathrm{~s}$ for the largest. When the approach was fast $(\ll 1 \mathrm{~s})$ the tip encountered the surface above its original height. Then, if the tip found the surface without a short circuit occurring immediately, the surface continued approaching its original height very slowly (tens of seconds). Alternatively, if a short circuit occurred immediately an even larger retraction was needed to break it. This resulted in a runaway situation in which successive retractions eventually spanned the entire retraction range.

We attribute the short circuit to a "jump" by the surface to contact with the tip, in response to attractive tipsurface interaction [Figs. 3(a) and 3(b)], which we speculate to be the van der Waals attraction. This would
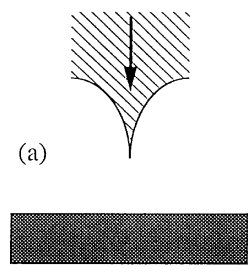

(c)

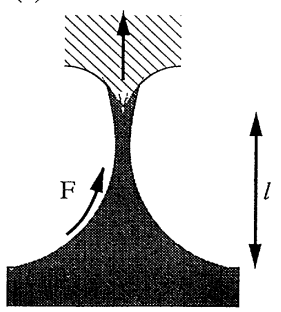

(b)

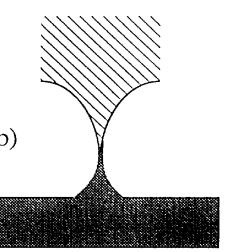

(d)

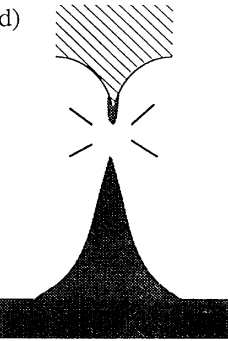

FIG. 3. Schematic cycle of the formation and rupture of surface-tip contact: (a) Approach of the tip. (b) Just after the jump to contact. (c) Growth of a neck. (d) Breaking of the neck. 
make the jump more probable when the tip-surface separation is reduced, due to the increase in the attraction and the reduction of the required jump distance, which explains why we observed regular tunneling at lower sample voltages (tip closer to the surface) for shorter times than at higher voltages. From the abrupt change in the measured current from zero to short circuit at the higher temperatures we conclude that the jump is made over a distance of at least $10 \AA$. The jump to contact results in a neck of $\mathrm{Pb}$ connecting the tip and the surface. As the tip is retracted the neck lengthens [Fig. 3(c)] and may eventually break [Fig. 3(d)]. The observed neck heights imply the relocation of several tens of millions of atoms during the buildup of these necks. After rupture the remaining hillock on the surface decays due to the surface tension. Thus the tip finds the surface again at approximately the same height where the jump occurred. The small dependence of the neck height on the short circuit current could be due to a local heating of the neck when it is on the verge of breaking.

We have observed this phenomenon on two different $\mathrm{Pb}(110)$ samples with two different tips on each sample, which strongly suggests that both the occurrence of the short circuit and its persistence were not an artifact, e.g., due to a peculiar tip shape. To rule out the possibility that the short circuits were a mere consequence of a sudden thermal expansion within the microscope, we calculated the thermal expansion of a tip in full thermal contact with the surface. The maximum expansion expected for a tip with a conical shape and a large contact diameter of $1 \mu \mathrm{m}$ amounts to only $400 \AA$ at the highest temperature in Fig. 2 of $382 \mathrm{~K}$. This is much smaller than the experimental retraction $(5000 \AA)$ at that temperature and it is even smaller than the minimum retraction measured at $318 \mathrm{~K}$. For more realistically tapered tips, the expansion would be a factor 2 to 4 smaller than that calculated for a conical tip. Additionally, the time constant calculated for the expansion of $400 \AA$ is $\approx 50 \mathrm{~s}$, corresponding to a speed of only $8 \AA / \mathrm{s}$. This is much slower than the minimum retraction speed of $100 \AA / \mathrm{s}$ used in the experiment. The electrical power dissipated in the neck is very low $(<1 \mathrm{pW})$ because of the reduced voltage drop over the junction. Thermal expansion due to this power can therefore be neglected. As a final argument against a trivial thermal effect, we stress that even at sample temperatures above the melting point of $\mathrm{Pb}$ our STM routinely images other metal surfaces, e.g., Au(110), with atomic resolution.

All the temperatures in Fig. 2 are below the temperature range in which the $\mathrm{Pb}(110)$ surface melts. This suggests that once the neck has reached an appreciable size, it is largely solid. We therefore assume that highly mobile $\mathrm{Pb}$ adatoms are responsible for the mass transport needed to build up the neck. As soon as the surface is unable to provide enough adatoms to follow the tip, the neck breaks. In order to estimate the activation energy for the formation of the neck we assume that the neck grows iso- morphously, so that local slopes on the surface remain constant and local curvatures $K$ are inversely proportional to the height of the neck $l$. The flux $F$ [Fig. 3(c)] of adatoms crossing a contour of constant $K$, then scales as

$$
F \propto D \nabla K \propto D / l^{2}
$$

Here, $D$ is the diffusion coefficient for mass transport, which combines the density of adatoms and their mobility. The growth of the neck slows down with the neck size as

$$
d l / d t \propto D \nabla^{2} K \propto D / l^{3} .
$$

If we assume that the neck breaks when the growth speed equals the tip-retraction speed $v$ we get

$$
L \propto(D / v)^{1 / 3},
$$

where $L$ is the height of the neck just before rupture. The diffusion coefficient varies with temperature as

$$
D=D_{0} \exp \left(-E_{\text {act }} / k_{B} T\right),
$$

where $k_{B}$ is the Boltzmann constant. This implies that $L$ should display Arrhenius behavior with an apparent activation energy of $E_{\text {act }} / 3$.

Figure 4 shows an Arrhenius plot of the rupture height for several retraction speeds. From this we obtain $E_{\text {act }}$ $=1.0 \pm 0.1 \mathrm{eV}$. This is close to the value for self-diffusion of adatoms on the same surface of $1.0 \pm 0.3 \mathrm{eV}$ found by He scattering [10]. Note that the activation energy for mass transport should be the sum of the energy for the creation of the adatoms and the activation energy for their diffusive motion, so that the value reported here should be higher than that in Ref. [10]. In reality, the growth of the neck is likely to be nonisomorphous and the curvatures should decrease more rapidly than $1 / l$. Using the above analysis we would then derive an activation en-

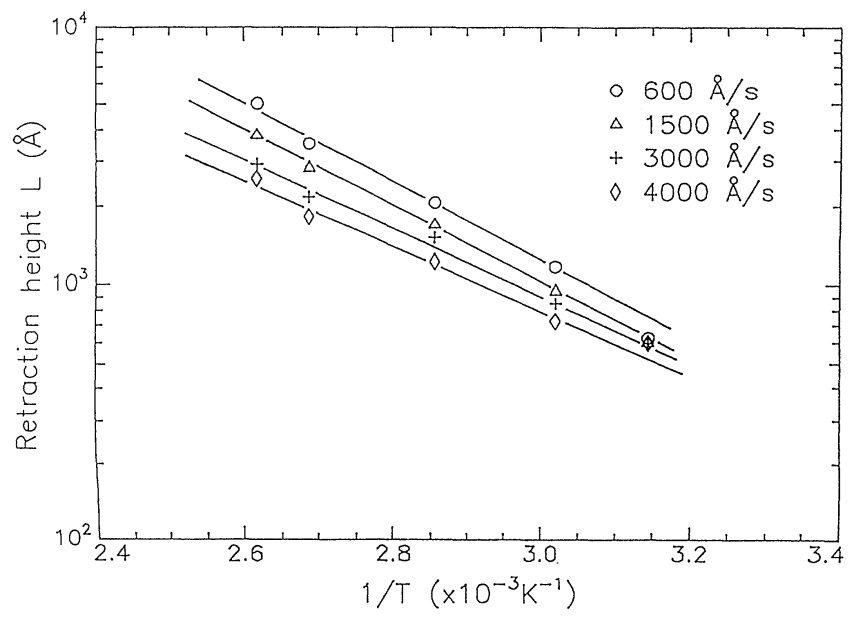

FIG. 4. Arrhenius plot of the retraction height at rupture for different tip-retraction speeds (initial sample voltage $-5 \mathrm{~V}$, short circuit current $23 \mathrm{nA}$ ). 
ergy larger than $1.0 \mathrm{eV}$. The slope in the Arrhenius plot depends somewhat on retraction speed. This cannot be explained by the simple isomorphous growth model and needs further investigation.

The present observations are very different from previous work [3-5] in two ways. First, we observe that the probability for the surface jumping to contact with the tip depends on temperature. Second, this study shows that massive necks can be formed instead of a stable point contact with a finite contact area. Recent computer simulations show that within a time window of a few tens of ps the $\mathrm{Pb}(110)$ surface jumps to contact with a $\mathrm{Au}$ AFM tip [8]. Even at room temperature a jump occurs when the tip is brought very close to the surface. When the surface melts a jump over large distances is observed together with the massive relocation of the surface atoms.

Finally, we refer to recent work by Züger and Dürig who have studied various surface orientations of Ga using a STM up to $0.1 \mathrm{~K}$ below the melting point of Ga [13]. The Ga surfaces remained ordered over the entire temperature range, and no jump to contact occurred. Close to the bulk melting point very few diffusion events were observed, indicating a low concentration of mobile adatoms. By contrast, we see many diffusion events on $\mathrm{Pb}(110)$, even at room temperature. Adatom-vacancy pairs are known to play an important role in both the melting of surfaces [14] and in the jump to contact [7]. Combining this information, we suggest that the strongly temperature-dependent jump to contact, and in particular the subsequent neck buildup, on $\mathrm{Pb}(110)$ are correlated with the disordering mechanism of this surface at higher temperatures.

The authors gratefully acknowledge a critical reading of the manuscript by J. S. Custer and stimulating discussions with $\mathrm{O}$. Tomagnini and $\mathrm{E}$. Tosatti who suggested this type of experiment. We thank A. J. Riemersma of the University of Amsterdam and R. J. I. M. Koper of our own institute for the preparation of our $\mathrm{Pb}$ sample. This work is part of the research program of the Foundation for Fundamental Research on Matter (FOM) and was made possible by financial support from the Netherlands Organisation for the Scientific Research (NWO).

[1] G. Binnig, H. Rohrer, Ch. Gerber, and A. Weibel, Phys. Rev. Lett. 49, 57 (1982).

[2] G. Binnig, C. F. Quate, and Ch. Gerber, Phys. Rev. Lett. 56, 930 (1986).

[3] J. B. Pethica and A. P. Sutton, J. Vac. Sci. Technol. A 6, 2490 (1988).

[4] J. K. Gimzewski and R. Möller, Phys. Rev. B 36, 1284 (1987).

[5] U. Landman, W. D. Luedtke, N. A. Burnham, and R. J. Colton, Science 248, 454 (1990).

[6] A. P. Sutton, J. B. Pethica, H. Rafii-Tabar, and J. A. Nieminen, in Electron Theory in Alloy Design, edited by D. G. Pettifor and A. H. Cottrell (Institute of Materials, London, 1992), p. 191.

[7] For jump to contact between planar surfaces, see R. M. Lynden-Bell, Surf. Sci. 244, 266 (1991).

[8] O. Tomagnini, F. Ercolessi, and E. Tosatti (to be published).

[9] J. F. van der Veen, B. Pluis, and A. W. Denier van der Gon, in Chemistry and Physics of Solid Surfaces, edited by R. Vanselow and R. F. Howe (Springer, Berlin, 1988), Vol. II, p. 455, and references therein.

[10] J. W. M. Frenken, B. J. Hinch, J. P. Toennies, and Ch. Wöll, Phys. Rev. B 41, 938 (1990).

[11] M. Poensgen, J. F. Wolf, J. Frohn, M. Giesen, and H. Ibach, Surf. Sci. 274, 430 (1992).

[12] J. W. M. Frenken, R. J. Hamers, and J. E. Demuth, J. Vac. Sci. Technol. A 8, 293 (1990).

[13] O. Züger and U. Dürig, Ultramicroscopy 42-44, 520 (1992).

[14] H. Häkkinen and M. Manninen, Phys. Rev. B 46, 1725 (1992), and references therein. 


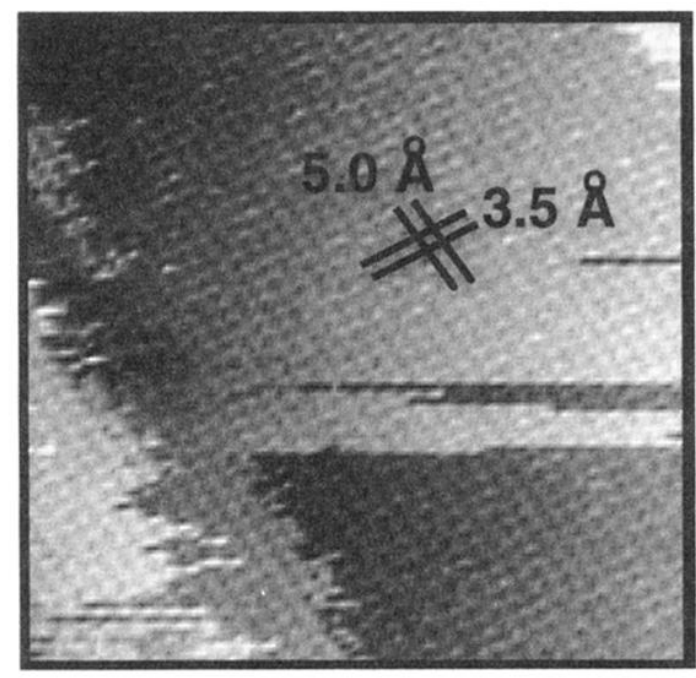

FIG. 1. Grey scale representation of an STM image of the $\mathrm{Pb}(110)$ surface at room temperature $(131 \AA \times 128 \AA$, $\left.V_{t}=+230 \mathrm{mV}, I_{t}=1.0 \mathrm{nA}\right)$. The grid indicates the $[1 \overline{1} 0]$ and the [001] directions. The numbers indicate the distance between atoms in angstroms. 